American Journal of Applied Sciences 9 (6): 784-793, 2012

ISSN 1546-9239

(C) 2012 Science Publications

\title{
Current and Future Nanotech Applications in the Oil Industry
}

\author{
${ }^{1}$ Cocuzza Matteo, ${ }^{1}$ Pirri Candido, ${ }^{2}$ Rocca Vera and ${ }^{2}$ Verga Francesca \\ ${ }^{1}$ Department of Materials, Science and Chemical Engineering, \\ ${ }^{2}$ Department of DIATI, Petroleum Engineering Group, \\ Engineering Faculty, Politecnico di Torino, \\ Corso Duca Degli Abruzzi 24, 10129 Torino, Italy
}

\begin{abstract}
Problem statement: Nanotech applications in the oil industry are not completely new: nanoparticles have been successfully used in drilling muds for the past 50 years. Only recently all the other key areas of the oil industry, such as exploration, primary and assisted production, monitoring, refining and distribution, are approaching nanotechnologies as the potential Philosopher's stone for facing critical issues related to remote locations (such as ultra-deep water and artic environments), harsh conditions (high-temperature and high-pressure formations), nonconventional reservoirs (heavy oils, tight gas, tar sands). The general aim is to bridge the gap between the oil industry and nanotechnology community using various initiatives such as consortia between oil and service companies and nanotechnology excellence centres, networking communities, workshops and conferences and even dedicated research units inside some oil companies. Quite surprisingly, even if a lot of discussion is taking place, no substantial research on these topics is currently being undertaken around the world by the petroleum industry. A very different attitude is demonstrated by other industries and the advances they achieved are outstanding. Approach: This study provides an overview of the most interesting nanotechnology applications and critically highlights the potential benefits that could come from transposing the same-or adapted-solutions to the oil industry. Results/Conclusion: As extensively illustrated, some technologies which are already available off-the-shelf can offer real improvements in dealing with some specific issues of the oil industry. Other technologies can require further elaboration before direct use, but their potential is enormous.
\end{abstract}

Key words: Department of Energy (DOE), Carbon Nano Tubes (CNTs), Magnetic Resonance Imaging (MRI), Advanced Energy Consortium (AEC), Single-Walled-Carbon-Nano Tubes (SWNT)

\section{INTRODUCTION}

According to well-established estimates by the U.S. Department of Energy (DOE), nowadays approximately $67 \%$ of the U.S. oil remains in place and it are generally known that the worldwide scenario is not much different, although figures might not be supported by as accurate global statistics. Furthermore, the dramatic decrease of easily recoverable oil and gas reserves and the existence of geopolitical tensions in those countries crucial for oil supply have triggered the necessity to explore unconventional reservoirs; at the same time, the environmental regulations currently enforced in most countries have imposed new practices and standards. As a consequence, the oil industry is facing important technical challenges, related to operating in deep and ultra-deep waters or in harsh conditions, to drilling and completing complicate wellbore profiles, to increasing the recovery factor in mature assets, to complying with safety issues. However, state-of-the-art technologies are still not able to fully satisfy the need to accurately locate and characterize the hydrocarbons in place in these new scenarios. With the exception of 3-D seismic surveys, the desirable resolution together with the ability to deeply penetrate the reservoir systems are not yet satisfied. Furthermore, the oil industry still relies on downhole electrical and electromagnetic imaging methods to improve the understanding of a reservoir (Kong and Ohadi, 2010), but conventional electrical sensors and other measuring tools may fail or be unreliable in hostile high temperature and high pressure conditions. Besides, the approaches currently available and used worldwide for enhanced oil recovery, such as thermal recovery, gas injection and chemical methods, have been partially hampered in different ways, either by relatively high costs or by relatively inefficient oil recovery. In conventional water and gas flooding, the

Corresponding Author:Rocca Vera, Department of DIATI, Petroleum Engineering Group, Engineering Faculty, Politecnico di Torino, Corso Duca Degli Abruzzi 24, 10129 Torino, Italy Tel: +39 0110907644 Fax: +39 0110907699 
injected fluids often quickly channel through the formation bypassing most of the oil in place due to unfavorable mobility ratios. Chemical EOR processes, such as polymer or surfactant flooding and alkaline injection, are also limited by high costs, potential corrosion of the formation and fluid loss during the flow through the reservoir (Kong and Ohadi, 2010).

Recently, the oil industry has been approaching nanotechnologies as a potential solution to the abovementioned challenges, calling for the same breakthrough effects that this relatively new branch of science has been gushing over the last 20 years in aerospace, biology and medicine. Properties of nanomaterials such as lightness, corrosion resistance and mechanical strength are and will be significant enablers, for example, for drilling and completion activities. Nanotechnology could also represent a breakthrough element for prospection, thanks to the development of innovative monitoring techniques and smarter micro/nano sensors. Other emerging applications of nanotechnology are represented by the development of new types of "smart fluids" for water shut-off and improved/enhanced oil recovery.

\section{MATERIALS AND METHODS}

Overview of present nanotech applications worldwide: Research in the nanoscience area and development of nanotechnologies are both moving extremely rapidly and their effects are now evident and well-established in many industrial divisions. The most disparate sectors, from health care to highly competitive and more industrially oriented compartments (automotive, aerospace, textile, energy and chemical processing,) have been looking at nanotechnology with growing expectation. The general aims are both to find possible solutions for never satisfied needs and to increase safety, quality and reliability of existing technologies at more favorable economic conditions.

A high percentage of nano-related "products" is represented by devices or structures in which top-down (i.e., miniaturized) technologies are employed to obtain electronic, fluidic or mechanical functions that can be applied to integrated circuits, sensors, telecommunications, environmental monitoring or biooriented diagnostics. As far as the top-down approach is concerned, nanotechnology is a natural continuation and improvement of the miniaturization revolution introduced with the microelectronic era. Nevertheless, the true nano-revolution relies on the full exploitation of the bottom-up approach, i.e., the creation of smart materials by exploiting their self-organisational capacity. The ultimate breakthrough of the nanotechnologies will be the extensive integration between the top-down and the bottom-up approaches: currently, very few serious plans about cooperation have been set forth, despite the numerous theorized roadmaps about convergence. Yet, there is a wide consensus that the future of nanotechnology lies in the integration of biological tools and systems into nanotechnological design and manufacturing.

Many companies are spending considerable financial resources in researching a specific area of nanotechnology called Carbon Nan Tubes (CNTs). Carbon nanotubes are made by carbon atoms forming hollow, open-ended cylinders that have a diameter between 0.4 and $1.8 \mathrm{~nm}$ and vary in length up to several hundred nanometers, depending on the production methodologies. Electrons flow through these nanotubes 10 times faster than they do through CPUs silicon circuits, they can carry up to 100 times the current and dissipate up to 20 times the heat. Carbon nanotubes can also act as either conducting or semiconducting material for use in data storage (Cui et al., 2002), while scanning probe microscopes may eventually be used as a tool for data transfer.

Another nanotechnology material, called graphene, has long been regarded as one of the most promising technologies to emerge. The latest development saw researchers at Samsung and Sungkyunkwan University in Korea produce a continuous layer of pure graphene which could be used to conduct electricity across flexible, transparent touchscreens based on a sheet of carbon just one-atom thick that can be folded like paper (Kim et al., 2009). The same material can also be used to make displays lighter and more power-efficient.

In the following a brief overview of the existing applications and near future improvement of nano-related "products" in different industrial sectors is presented and discussed with the aim of underlying similarities and thus possible applications to the oil industry.

Medicine and biology: Medicine and biology are surely among the most promising, but at the same time most challenging, fields of application for nanotechnologies; and the medical field is probably the sector where nanotechnologies are currently introducing the most exciting innovations. The genesis of nanomedicine sprang from the idea that devices could be designed, manufactured and introduced into the human body to perform cellular and tissue repairs (Drexler, 1986; Drexler et al., 1991; Freitas, 2000; Freitas, 2003). A variety of different types of nanoobjects are being experimented (or already commercialized), including carbon nanotubes, other carbon-based 'skeleton structures' such as 'bucky balls' or fullerenes, dendrimers, drug carriers, nanoparticles for scanning, nanobarcodes, nanoemulsions, nanofibres, 
nanoshells, nanocolloids, magnetic and superparamagnetic beads and quantum dots.

Nanotechnology enables delivery and release of drugs to a specific location in the body with specified timing to provide the most effective treatment. This is achieved by fixing the drug to a tiny carrier (silica or gold-coated nanoshells) which has been pre-treated to seek for a specific area of the body and bind to it. Drugs will be released when the shell reaches the proper location and is melted by the heat generated with infrared (Sershen et al., 2000) or radio frequency stimulation.

Nanoparticles with special magnetic properties are being considered for cancer treatment. Injected into the patient's body and guided to tumour cells, these nanoparticles will develop heat when exposed to high frequency electromagnetic fields. The technology and its related equipment are currently in the final phase of clinical trials (Magforce website, http://www.magforce.de).

Nanoparticles are also used to obtain better quality images with several imaging methods currently in use, such as computed tomography scans (CT scans), Magnetic Resonance Imaging (MRI) and ultrasound imaging. The way in which nanoparticles help is largely by improving the contrast in the images.

Another extremely challenging field is that of tissue engineering, an interdisciplinary biology and engineering approach with the aim of artificial substitution, or restoration and improvement of human tissue functions (such as: nerve regeneration and interfacing, development of bio-artificial organs, regrowth of bones and vessels, substitution of skin and sensory organs). As far as artificial sensory organ development is concerned, nanotechnologies play a key role not only in artificial sensor design and fabrication, but also in the signal processing and the neuroelectronic stimulation techniques to drive nerve fibers and cells that are no longer naturally stimulated. In this framework, it is currently possible to provide some sort of visual sense to blind people. Eyesight can be partly restored thanks to implantable epi-retinal microchips designed to electrically stimulate the visual system at multiple points and create a sense of vision. The next generation device, due by 2012 , will likely provide the ability to read and the final goal is to restore most of the abilities of normal sight.

Automotive industry: A totally different and extremely competitive sector that is appreciating what nanotechnology can offer is the automotive industry. Also in this case, the lion's part is played by innovation in materials at the nanoscale. Lightweight but rugged polymer nanocomposites are extensively used for body parts, while new metal nanocomposites are employed to improve engine efficiency Plastic Technology Website.
To account for the more and more stringent safety and environmental regulations, specially customised nanoparticles are presently used as fuel additives to reduce fuel consumption and toxic emissions. Furthermore, the on-going trend is the integration of smart nano-based sensors for prevention of collisions, improved navigation systems and prevention of possible driving difficulties due to tire blow-out (Fleming, 2001; Valldorf and Gessner, 2005). Improved and more efficient end-of-life recycling procedures will also take advantage of the innovative new materials introduced in the car design and production chain.

Environmental sustainability: Nanotechnology offers some really exciting breakthroughs in environmentally friendly technologies, from renewable energy sources to the prevention of pollution. Today, more efficient and cheaper solar energy collectors using nanostructured materials are on their way. A further development of solar cells deals with the simulation of the chlorophyll plant process thanks to nanomaterials (Wang et al., 2005).

Energy conversion is the sector of fuel cells (Yamamoto et al., 2009), where the nanotechnology potential lies in the core of the device, i.e., the membrane, which is responsible for the separation of hydrogen into protons and electrons. For energy storage, nanotechnology embodies a powerful tool for safely storing the hydrogen inside nanostructures such as carbon nanotubes (Berseth et al., 2009), zeolithicbased materials (Weitkamp et al., 1995) and nanodusts. Furthermore, nanotechnology is leading the development of more efficient and environmentally friendly batteries and supercapacitors, with increased available power and decreased recharging time. Generally, these benefits are achieved by coating the electrodes surfaces with nanoparticles. Because of the consequent surface increment, more electricity can flow between the electrodes and the chemicals inside the battery (Manev, 2009). Nanotechnology is also leading to eradication of toxic solvent chemicals through the development of new nanocoatings and nanostructured surfaces.

Textile industry: Although major breakthroughs from nanotechnologies were expected in fields such as medicine and electronics, the textile industry has been one of the initial adopters of nanotechnology products and processes, both for the development of new materials and for process innovation to reach lower unit costs, larger capacity and better quality. Two main target areas could be identified: upgrading current functions and performances of textile materials, mainly through manufacturing of composite fibres utilizing nanosize fillers such as nanoparticles (Soane et al., 
Am. J. Applied Sci., 9 (6): 784-793, 2012

2003) and carbon nanotubes (Zhang et al., 2005), or developing improved chemical finishes with outstanding stain-resistant and anti-static (Nano-Tex website, http://www.nano-tex.com), hydrophilic (Naor et al., 2006) and shrink-proof features.

\section{RESULTS}

Overview of present nanotech applications in the oil industry: Currently, the general aim is to bridge the gap between the oil industry and the nanotechnology community, as highlighted by the increasing number of related social and technical events, such as dedicated SPE workshops, specialized web communities (i.e., Nano-E\&P Network) and, above all, by research projects arisen from the collaboration among major oil companies and academia or excellence research institutes.

In 2008, with the purpose of exploring the potential of nanotechnology, the Advanced Energy Consortium (AEC) was constituted in the U.S., in cooperation with most of the major oil and service companies, such as BP, ConocoPhillips, Shell, Total and Schlumberger. This consortium, with an annual budget of several billion dollars, was given the task of exploiting nanotechnologies to improve oil and gas production. The primary goal is to develop subsurface micro- and nanosensors that could be injected in oil and gas well bores (Kong and Ohadi, 2010).

A great number of researchers are also focused on the identification of nanotech potentialities applied to EOR issues. As an example, the Oklahoma University, supported by AEC, has already published results about Single-Walled-Carbon-Nano Tube (SWNT)/Silica nanohybrids, active at the fluid interface and thus potentially suited for EOR. These particles represent very promising materials: if delivered at the $\mathrm{O} / \mathrm{W}$ interface, they would react with and modify the oil properties to increase oil mobilization.

Oil industries are also beginning to play an active role in the cooperation between private (especially medical and aerospace industries) and public (such as the US Department of Defence, NASA,) sectors in supporting early stage technology companies during the development and the adjustment of innovative nanoenhanced materials and advanced coating cladding technology. For example, the research on nanotubes by NanoRidge has received funding from major corporations in oil and aerospace (a key market for higher performance materials) as well as federal research support including a grant from the Research Partnership to Secure Energy for America (Rassenfoss, 2011).
At the moment, the research is analysing the potential benefits derived by the application of nanomaterials, nanofluids and nanomembranes to the oil industry. In the following a more detailed overview of the recent progresses in research and application in key oil and gas sectors is provided.

Sensors: According to Krishnamoorti (2006), nanomaterials are excellent tools for the development of sensors and imaging-contrast agents due to the significant alterations in their optical, magnetic and electrical properties (in comparison to their bulk analogues) along with their ability to form (electrically and/or geometrically) percolated structures at low volume fractions. Such nanomaterials, when combined with smart fluids, can be used as extremely sensitive downhole sensors for temperature, pressure and stress even under extreme conditions. The ultimate evolution of devices for prospection is represented by nanorobots, which should really provide an effective mapping of the reservoir. Nowadays, nanorobots still remain a dream, shared by the medical and oil sectors. But advances in nanosensor miniaturization are occurring rapidly and numerous theoretical and experimental investigations about the flow of multiphase fluids containing nanoparticles in porous media enrich the recent technical literature (Ryoo et al., 2010; Yu et al., 2010).

Coatings: Significant work is underway toward the transition of smart/multifunctional polymer coatings from laboratory curiosities toward the identification of commercial applications. Intelligent or smart coatings, which may combine the shielding aspect with sensor or actuator functions, rely on their capabilities to respond to physical, chemical or mechanical stimuli by developing readable signals. Nanomaterials are expected to be used not only as advanced functional materials, but also as an integral part of complete smart structures composed of various elements including sensors, actuators, control devices.

Some of the key challenges in more advanced research areas are the understanding of corrosion protection mechanism imparted by conducting polymers and the advancement of micro/nanocapsulation as a means to impart selfhealing (Boura et al., 2010). Nevertheless, some innovative applications seem to be ready for commercialization in a very nearby future, such as a coating using carbon nanotubes to conduct a current for evenly heating surface, which could be used on pipelines to reduce gas hydrate formation or to de-ice the blades on wind turbines (Rassenfoss, 2011). 
An innovative corrosion-resistant material solution could also be represented by nanometric thin films and composites with nanostructured fillers. Apart from the economic aspect, which is not strongly favorable yet, corrosion-resistant materials are surely the "just round the corner" nanotechnology-based applications, basically because of the combination of several conditions: relatively low risk, high effectiveness and low complexity. Nano-coated, wear-resistant probes, made of tungsten carbide or boron nitride, enhance the lifespan and efficiency of the drilling systems, thus inducing remarkable cost savings. The same applies to the nano-layered corrosion inhibitors in pipes or tanks, which act through the creation of a permanent molecular layer on the surface of metals, thus eliminating or hampering corrosion induced by $\mathrm{HCl}$ or $\mathrm{H}_{2} \mathrm{~S}$.

Nanofluids and nanomaterials for drilling and completion: Drilling and completion sectors are other two oil branches where the benefits of nanofluids and nanomaterials application are already tangible. Nanotechnology has opened the door to the development of a new generation of fluids defined as "smart fluids" for drilling, production and stimulationrelated applications. Thanks to the exceptionally high surface to volume ratio, nanofluids and nano-based additives exhibit major interaction with the surrounding environment even at very low concentrations. Such smart fluids will further enhance drilling by adding benefits such as wettability alteration, advanced drag reduction and sand consolidation (Chaaudhury, 2003; Wasan and Nikolov, 2003).

One specialized petroleum laboratory has developed an advanced fluid mixed with nanosized particles and superfine powder that significantly improves the drilling speed and can eliminate formation damage in near wellbore zone (Esmaeili, 2009).

Prof. Tour's Laboratory works with M-I SWACO's to optimize the effectiveness of graphene additives to drilling fluids Nanowerk Website, 2009, Rice University News and Media Relations Website, 2009.

Thanks to the synthesis of a new class of elastomeric composites filled with carbon nanotubes or other strongly anisotropic nano-objects, stronger, tougher and more resistant drilling tools and apparatus will be manufactured in the coming years. At the same time, these tools will ensure a significant weight reduction and the potential to originate self-sensing elements to be interrogated for the real-time monitoring of the most critical parts.

Another important technique in the development of super-hard materials is the use of nanostructured dispersed-hardened materials (Terranova et al., 1999). The superiority of physical-mechanical properties of diamond polycrystalline nanocomposites (Terranova et al., 1999), boron nitride nanocomposites (Dubrovinskaia et al., 2007) and 2WC/Co/diamond nanocomposites (Jain, 2001) in comparison with their traditional counterparts has been reported in the literature.

First generation of nanotech applications for improving hydraulic fracturing are represented by Baker Hughes's nano-structured metal composites, combined by magnesium, aluminium and other alloys, which offer both strength at lower weight and the ability to "dissolve" away under certain conditions. Another example is the proppant produced by Oxane Materials, constituted by nano-structured ceramic material which is as strong as but lighter as ceramic proppant.

A possible solution for mitigating fine migration problems is represented by the commercialized nanocrystals for treating hydraulic fracture proppant packs to fixate formation fines. The mechanism of fixation of the formation fines depends on the high surface forces of the nanoparticles, such as Van der Waals and electrostatic forces, which also attach the nanoparticles to the surface of proppant during fracpacking and fracturing treatments (Huang et al., 2008).

Nanomembranes: Inspired by the success of zeolites, which are materials capable of separating small gases such as oxygen and nitrogen, a new generation of largescale, lightweight and sturdy nanomembranes is being developed and deployed. These nanomembranes will significantly enhance the exploitation of tight gas by providing efficient methods for removing impurities, separating gas streams and enabling GTL production. By exploiting methods common in the microelectronics industry, the cost of manufacturing highly uniform and reproducible membranes is quite competitive (Krishnamoorti, 2006). Nanoporous and nanoparticular materials are also very promising to manage the environmental, health and safety risks deriving from the presence of $\mathrm{CO}_{2}$ and $\mathrm{H}_{2} \mathrm{~S}$ in hydrocarbon mixtures.

\section{DISCUSSION}

Evolution of nanotechnology application for reservoir monitoring: The petroleum sector and the medical one share the same dream of a nanosensor able to provide direct (in-situ) and reliable information about the system under analyses. Nowadays, this represents one of the most complicated and transversal challenge faced by oil industry because it requires strong technical improvements in many different disciplines. In fact, the exploitation of injected nanosensors into the reservoir is subject to the satisfaction of the following technological requirements: emplacement and recovery, protection in harsh environments (through a proper shell or coating), 3-D location, sensors powering, data storage and retrieving. 
The current, most advanced, commercial device which somewhat embodies the idea of the miniaturised submarine is the swallowable capsule developed for medical purposes (Johannessen et al., 2004). It is an electronic-based microsystem which can be ingested to explore the gastrointestinal tract and transmit the acquired information to a base station. It is a complete system, with sensors that convert physical properties such as light, pressure, or temperature into electrical signals, while actuators perform the opposite function. The signal-conditioning block provides analog processing such as amplification and filtering to "clean" the detected signal. The system's brain, the CPU, digitizes the signal and might perform additional processing. The communication block can then transmit the signal to a receiver module outside the body. The communication medium can be RF, a magnetic field (inductive coupling), or ultrasound. Finally, the power supply, based on either batteries or inductive coupling, provides energy for the system. Unfortunately, this existing technology cannot be directly transferred to the oil industry due to issues which today technology is not still ready to solve, such as the 3-D location of the sensors. Above all, the swallowable capsule is several millimetres wide and long, thus absolutely not compatible with the pore throat dimensions of the reservoir rocks. This confirms that the direct investigation and monitoring of reservoirs with miniaturized instruments remains a long-term task. According to the authors' opinion, a reasonable way forward would be to decompose the problem in the improvement of each single sub-component of the whole ideal system, such as: scaffold, power unit, communication unit, sensing elements, data storage and management, motion and position control and nanosystem retrieving. By this approach the final goal could be achieved progressively, through the development of smarter and smarter nanosensors.

Based on the available technology the path to "slightly" smart nanosensors is shorter and could introduce significant advantages for reservoir investigation. 100-1000 nm diameter passive nanoobjects could be flushed with the injection fluids through the pores of the reservoir rocks to determine the formation characteristics. No active components (sensor, data storage or transmission, 3D location, power) would be on-board, but the presence of a proper structure (multi-wall nanowires, core-shell particles,) interacting with the reservoir could retrieve threshold information (maximum temperature and/or pressure, maximum $\mathrm{pH}$, salinity,). The magnetic (through a magnetic core) or electrical (as in the case of Carbon nanotubes) conductivity of such nano-dust could be exploited for recovering information. Using a core-shell structure, for example, the quantity of oil present in a reservoir could be assessed based on the amount of material lost or retained during the travel time, or the extreme conditions (temperature, pressure, salinity gradient) at which the nanoparticles were exposed and for how long, could be determined. The idea could be to pump nano-sensors in the reservoir periodically so as to regularly monitor changes in the well/field conditions. In turn, this could result in improved production efficiency and trouble managing (Durham, 2009). An interesting and extremely efficient property which could be exploited at the nanoscale is the shape memory effect. Nanoparticles based on specific alloys or polymeric composites maximize the efficiency of the shape memory phenomenon, with the triggering of the shape change occurring as a result of changes in temperature, moisture intake or $\mathrm{pH}$ (Cook et al., 2007).

The subsequent improvement should be the nanosensors or nano-sensors clusters localization. Under this respect, the special electrical, optical and magnetic properties of nano-materials make them well suited for use as injected sensors and contrast agents. Several possible applications and exploitation schemes are currently under study with nano-devices injected into a reservoir. The position of the nano-devices could be measured at predesigned time delays by suitable crosswell or surface-based electro-magnetic procedures or seismic methods in the case of nano-devices behaving like acoustic pingers. As an example, in a project funded by the AEC Barron incorporated novel paramagnetic nanoparticles into proppant structures (Esmaeili, 2009): this smart proppant could help in mapping the fracture efficiency using detectable contrast agents. Furthermore, through a proper surface functionalization, nanoparticles could preferably segregate into different fluid portions, thus providing enhanced characterization of the reservoir and, as a consequence, improved oil recovery. Thanks to their small dimensions, increased surface area and high mobility, the use of such nanoparticles is very promising.

Several innovative materials and solutions could support the development of nanosensors. For example, as far as the scaffold is concerned, organic building materials (e.g., proteins, polynucleotides) are very good at self-assembly, but the most reliable and highperformance molecular machines may be constructed out of diamondoid materials, the strongest known substances. Building diamondoid nano-robots will require both massive parallelism in molecular fabrication and assembly processes and programmable positional assembly, including molecularly precise manufacture of diamond structures using molecular feedstock (Peng et al., 2004). Positionally controlled single-atom covalent bonding (mechano-synthesis) has been achieved experimentally for hydrogen and silicon 
Am. J. Applied Sci., 9 (6): 784-793, 2012

atoms (Oyabu et al., 2003), but presently only computational simulations support the same expectation for carbon atoms and diamond structures.

As far as the power is concerned, many nano-based tools are currently under development. Several options exist: a nano-battery operated system, a system able to scavenge the necessary energy in situ or a mixed solution. This last alternative is even more intriguing and some encouraging results have been obtained, particularly for the biomedical field. Researchers at the Georgia Tech are working on a method that could lead to medical devices that are powered by the patient's heartbeat. Wang and his team have deposited $\mathrm{ZnO}$ nanowires on a flexible polymer substrate; after that, the device was put into a polymer casing to protect it from body fluids interacting with it and was attached to the diaphragm of a laboratory rat (Wang and Song, 2006). It is possible to imagine that a forest of $\mathrm{ZnO}$ nanowires grafted on to the external surface of a diamondoid scaffold travelling into the reservoir matrix could generate electricity when bent because of the contact against the pore walls.

Evolution of nanotechnology application for enhanced oil recovery: The greatest challenge is concerned with the possibility of increasing the efficiency of hydrocarbon production, both by improving the current EOR methodologies and by developing alternative technologies. According to current researches and technical literature, the newgeneration nano-agents should both affect the properties of the injected fluid, in terms of viscosity, density, thermal conductivity and specific heat and modify the fluid-rock interaction properties, for example in terms of wettability.

Reports indicate the use of two different types of polysilicon nanoparticles in oil fields to enhance water injection and improve oil recovery ( $\mathrm{Ju}$ and $\mathrm{Fa}, 2009$ ). Reportedly, one nanometer-scale polysilicon material could change the wettability of porous surfaces of sandstone and consequently affect the flow of water and oil when injecting a suspension of nanoparticles in an oil reservoir (Ju and Dai, 2002).

Great interest sparked by the application of paramagnetic nanoparticles for EOR issues. According to (Kothari et al., 2010), ferrofluids possess a unique combination of fluidity and capability to interact with a magnetic field. The addition of a surfactant would greatly reduce the interfacial tension in water-wet reservoirs, so ferrofluids can shatter the oil globules forming in the reservoir. Also, as the ferrofluid comes in contact with the crude in the reservoir, it interacts with it: due to the presence of a dipole moment, the reservoir fluid molecules align, thus reducing the resistance to flow.
The University of Kansas, sponsored by ConocoPhillips, aims at creating a new class of polymer-type nanoparticles that can be incorporated with EOR injection fluids to improve hydrocarbon recovery. With their ultra-small size and very high surface area/volume ratios, nano-polymers can penetrate small pore throats without being trapped and the amount of expensive injection can be decreased (JPT online website, http://www.jptonline.org).

The University of Alaska Fairbanks investigated the possibility of improving gas injection for the Alaska North Slope heavy oil recovery thanks to metal nanoparticles. The research was focused on the following points (Shah, 2009): reduce the viscosity of the heavy oil using super critical $\mathrm{CO}_{2}$ and Viscosity Reducing Injectant (VRI) saturated by nanoparticles; exploit the thermal properties of nanoparticles for enhanced heat transfer to heavy oil; explore the effects of Poly Di Methyl Siloxane and metal nanoparticles on density and viscosity for reducing mobility and viscous fingering phenomena; study EOR processes on Berea sandstone cores, saturated with Alaska North Slope heavy oil, using $\mathrm{CO}_{2}$ and $\mathrm{CO}_{2}$ nanofluid gas core flood experiment.

When dealing with extremely low permeability reservoirs, conventional approaches to water flooding cannot provide recovery factors greater than 0.2 . This is mainly due to capillary forces which are very strong in narrow pore channels. With low-permeability reservoir rocks, the level of purity and filtration of injected water is one of the most important issues for a significant oil recovery. With rocks characterized by a permeability of $0.1 \mu \mathrm{m}^{2}$ the recovery factor deviates from an average of 0.4 by less than $10 \%$ if particulates in injected water are smaller than $200 \mathrm{~nm}$. Nevertheless, with a rock permeability of $0.05 \mu \mathrm{m}^{2}$ the recovery factor is dramatically approaching zero with the same particles size. A traditional water flooding technique is obviously unproductive in this latter case, unless water is filtered and purified of particulates down to at least $100 \mathrm{~nm}$. Innovative water nano-filters have to be specially designed both to ensure uniform pore throats and the ability to recovery tens of billions of tons of oil; this aspect is also very important, since the flow capacity of currently employed biomedical filters would not be adequate for the purpose. To provide an evidence of the impact of such a nanotechnological breakthrough, it should be considered that the reservoir rocks of the Bazhenov formation (the main hydrocarbons reservoirs in Russia) are characterized by a mean pore radius of 1-10 $\mathrm{nm}$, which is comparable with molecular sizes (Ya, 2009).

More generally, for the specific aim of EOR applications, some authors underline that oil is essentially a nanoliquid, with asphaltenes being the dominant part, flowing in a micro and often nanoscopic environment (i.e., the porous media). According to that, 
it seems justifiable to consider most of the oil and gas production technologies, reservoir treatments and stimulation as nanotechnologies (Evdokimov et al., 2006). Under this point of view, current petroleum science is relying on the study and control of physicchemical phenomena occurring at the nanometer scale and the ability to correctly model the reservoir flow plays an important role. However, a suitable tool to reliably describe coupled phenomena is still missing. Therefore, the construction of mathematical models for EOR technologies employing mechanical, thermal and electromagnetic "agents" should be considered a main task for the future applications of nanotechnologies to EOR.

With a long-term perspective, the idea is currently being discussed of creating nanorobots able to monitor individual pores and channels and hydrocarbon flow, exchange information and identify zones in which intensify the extraction or the injection of water or other oil recovery stimulating agents (Bhat and Singh, 2006).

\section{CONCLUSION}

The benefits that nanotechnology can offer to the oil industry are potentially enormous. As extensively illustrated in the study, some nanotechnology applications are already available to the market while others could come from transposing the solutions developed for the biomedical, automotive, aerospace, chemical and even textile sectors, where major breakthrough occurred thanks to the nanotechnology revolution. Well drilling, fracturing and cementing as well as new generation membranes for gas separation can already rely on nanotech solutions. Other technologies will require further elaboration before direct use. Currently, relevant efforts are being made to design nano-sensors for reservoir characterization and monitoring and to produce nano-fluids for improving EOR processes. Very promising results have been obtained from laboratory experiment, but field tests are still extremely limited.

The future of nanotechnology seems to be bright. Nevertheless, several issues are to be considered and the following actions should be taken to transform a big opportunity into reality: favour multi-disciplinarity, improve convergence between the top-down and the bottom-up approaches (namely, miniaturization and the creation of smart materials by exploiting their selforganisational capacity), be careful with the "nano" hype (often nano erroneously comprises traditional physics and chemistry) and, finally, consider the usual long-term research and investment time frame for targeting business properly.

\section{REFERENCES}

Berseth, P.A., A.G. Harter, R. Zidan, A. Blomqvist and C.M. Araujo et al., 2009. Carbon nanomaterials as catalysts for hydrogen uptake and release in NaAlH4. Nano Lett., 9: 1501-1505. DOI: 10.1021/n1803498e

Bhat, S and P. Singh, 2006. Nanologging: Use of Nanorobots for Logging. SPE Eastern Regional Meeting, Canton, Ohio, USA, Oct 11-13. DOI: 10.2118/104280-MS

Boura, S.H., M. Samadzadeh, M. Peikari and A. Ashrafi, 2010. Smart and multi-functional coatings based on Micro/Nano sized additives and their implementation. Proceedings of the SPE International Conference on Oilfield Corrosion, Aberdeen, United Kingdom, pp: 24-25. DOI: 10.2118/130972-MS

Chaaudhury, M.K., 2003. Complex fluids: Spread the word about nanofluids. Nature, 423: 131-132. DOI: $10.1038 / 423131 \mathrm{a}$

Cook, F.L., K.I. Jacob, M. Polk and B. Pourrsdeyhimi, 2007. Shape memory polymer fibers for comfort wear. NTC Project M05-GT14.

Cui, J.B., R. Sordan, M. Burghard and K. Kern, 2002. Carbon nanotube memory devices of high charge storage stability. Applied Phys. Lett., 81: 32603262. DOI: $10.1063 / 1.1516633$

Drexler, K.E., 1986. Engines of Creation. 1st Edn., Anchor Press/Doubleday, New York, ISBN: 0385199732, pp: 298.

Drexler, K.E., C. Peterson and G. Pergamit, 1991. Unbounding the Future: The Nanotechnology Revolution. 1st Edn., William Morrow/Quill Books, New York, ISBN: 0688125735, pp: 366.

Dubrovinskaia, N., V.L. Solozhenko, N. Miyajima, V. Dmitriev and O.O. Kurakevych et al., 2007. Superhard nanocomposite of dense polymorphs of boron nitride: Noncarbon material has reached diamond hardness. Applied Phys. Lett., 90. DOI: 10.1063/1.2711277

Durham, L.S., 2009. Researchers are thinking small. AAPG Explorer.

Esmaeili, A., 2009. Applications of nanotechnology in oil and gas industry. Proceedings of the 2nd International Conference on Methods and Models in Science and Technology, Nov. 19-20, AIP, Jaipur, India pp: 133-136. DOI: 10.1063/1.3669944

Evdokimov I.N., N.Yu. Eliseev, A.P. Losev, M.A. Novikov, 2006. Emerging petroleum-oriented nanotechnologies for reservoir engineering. Proceedings of the SPE Russian Oil and Gas Technical Conference and Exhibition 2006, Moscow, Russia, Oct. 3-6, Moscow, Russia, p: 370-378. DOI: $10.2118 / 102060-\mathrm{MS}$ 
Fleming, W.J., 2001. Overview of automotive sensors. IEEE Sensors J., 1: 296-308. DOI: 10.1109/7361.983469

Freitas, R.A., 2000. Nanodentistry. J. Am. Dent Assoc., 131: 1559-1566. PMID: 11103574

Freitas, R.A., 2003. Nanomedicine: Biocompatibility. 1st Edn., S Karger Ag, Basel, ISBN-10: 3805577222, pp: 348.

Huang, T.T., J.B. Crews and J.R. Willingham, 2008. Using nanoparticle technology to control fine migration. Proceedings of the SPE Annual Technical Conference and Exibition, Sep. 21-24, Denver, Colorado, USA., pp: 21-24. DOI: 10.2118/115384-MS

Jain, M., 2001. Processing of functionally graded WC/Co/diamond nanocomposites. Scripta Mater., 44: 8-9. DOI: 10.1016/S1359-6462(01)00882-X

Johannessen, E.A., L. Wang, L. Cui, T.B. Tang and M. Ahmadian et al., 2004. Implementation of multichannel sensors for remote biomedical measurements in a Microsystems format. IEEE Tran. Biomed. Eng., 51: 525-535. DOI: 10.1109/TBME.2003.820370

Journal of Petroleum Technology. monthly publication of the Society of Petroleum Engineers JPT online website,

Ju, B and S.G. Dai, 2002. A study of wettability and permeability change caused by adsorption of nanometer structured polysilicon on the surface of porous media. Proceedings of the SPE Asia Pacific Oil and Gas Conference and Exhibition, Oct. 8-10, Melbourne, Australia, pp: 8-10. DOI: 10.2118/77938-MS

$\mathrm{Ju}$, B. and T. Fa, 2009. Experimental study and mathematical model of nanoparticle transport in porous media. Powder Technol., 192: 195-202. DOI: 10.1016/j.powtec.2008.12.017

Kim, K.S., Y. Zhao, H. Jang, S.Y. Lee and J.M. Kim et al., 2009. Large-scale pattern growth of graphene films for stretchable transparent electrodes. Nature, 457: 706-710. DOI: 10.1038/nature07719

Kong, X. and M.M. Ohadi, 2010. Applications of micro and nano technologies in the oil and gas industry overview of the recent progress. Proceedings of the Abu Dhabi International Petroleum Exhibition Conference, Nov. 1-4, Abu Dhabi, UAE, pp: 1-4. DOI: 10.2118/138241-MS

Kothari, N., B. Raina, K. Chandak, V. Iyer and H. Mahajan, 2010. Application of ferrofluids for enhanced surfactant flooding in IOR. Proceedings of the SPE EUROPEC/EAGE Annual Conference and Exhibition, Jun. 14-17, Barcelona, Spain, pp: 14-17. DOI: 10.2118/131272-MS
Krishnamoorti, R., 2006. Extracting the benefits of nanotechnology for the oil industry. J. Petroleum Technol.

Manev, V., 2009. Methods for improving lithium ion battery safety. Altairnano Inc., United States Patent.

Naor, Y., Y. Barak and M. Mois, 2006. Moisturemanagement in hydrophilic fibers. Delta Galil Industries Ltd., World Patent.

Oyabu, N., O. Custance, I. Yi, Y. Sugawara and S. Morita, 2003. Mechanical vertical manipulation of selected single atoms by soft nanoindentation using near contact atomic force microscopy. Phys. Rev. Lett., 90: 176102. PMID: 12786084

Peng, J., R.A. Freitas and R.C. Merkle, 2004. Theoretical analysis of diamond mechanosynthesis. Part I. stability of $\mathrm{C} 2$ mediated growth of nanocrystalline diamond $\mathrm{C}(110)$ surface. J. Comput. Theoretical Nanosci., 1: 62-70. DOI: 10.1166/jctn.2004.007

Rassenfoss, S., 2011. Nanotechnology for sale: The oncetheoretical becomes pratical. J. Petroleum Technol.

Ryoo, S., R.A. Rahmani, Y.K. Yoon, M. Prodanović and C. Kotsmar et al., 2010. Theoretical and experimental investigation of the motion of multiphase fluids containing paramagnetic nanoparticles in porous media. Proceedings of the SPE Annual Technical Conference and Exhibition, Sep. 19-22, Florence, Italy, pp: 19-22. DOI: 10.2118/134879-MS

Sershen, S.R., S.L. Westcott, N.J. Halas and J.L. West, 2000. Temperature-sensitive polymer-nanoshell composite for photothermally modulated drug delivery. J. Biomed. Mater. Res., 51: 293-298. DOI: 10.1002/1097-4636(20000905)51:3<293::AIDJBM1>3.0.CO;2-T

Shah, R.D., 2009. Application of nanoparticle saturated injectant gases for EOR of heavy oils. Proceedings of the SPE Annual Technical Conference and Exhibition, Oct. 4-7, New Orleans, Luisiana, USA.

Soane, S., D.A. Offord, M.R. Linford, D.B. Millward and W. Ware et al., 2003. Nanoparticle-based permanent treatments for textiles. Nano-Tex, LLC, United States.

Terranova, M.L., S. Piccirillo, V. Sessa, M. Rossi and S. Botti, 1999. Microstructure and properties of nanocomposite diamond films obtained by a new CVD-based technique. J. Phys. IV France, 9: 365371. DOI: 10.1051/jp4:1999845

Valldorf, J. and W. Gessner, 2005. Advanced Microsystems for Automotive Applications. 1st Edn., Springer, Berlin, New York, ISBN: 3540244107, pp: 543. 
Wang, X.F., J. Xiang, P. Wang, Y. Koyama and S. Yanagida et al., 2005. Dye-sensitized solar cells using a chlorophyll a derivative as the sensitizer and carotenoids having different conjugation lengths as redox spacers. Chem. Phys. Lett., 408: 409-414. DOI: 10.1016/j.cplett.2005.04.067

Wang, Z.L and J. Song, 2006. Piezoelectric nanogenerators based on Zinc Oxide nanowire arrays. Science, 312: 242-246. DOI: 10.1126/science. 1124005

Wasan, D.T and A.D. Nikolov, 2003. Spreading of nanofluids on solids. Nature, 423: 156-159. DOI: 10.1038/nature01591

Weitkamp, J., M. Fritz and S. Ernst, 1995. Zeolites as media for hydrogen storage. Int. J. Hydrogen Energy, 20: 967-970. DOI: 10.1016/03603199(95)00058-L
Yamamoto, K., T. Imaoka, W.J. Chun, O. Enoki and H. Katoh et al., 2009. Size-specific catalytic activity of platinum clusters enhances oxygen reduction reactions. Nature Chem., 1: 397-402. DOI: 10.1038/nchem. 288

Yu, J., M.J. Berlin, W. Lu, L. Zhang and T.A. Kan et al., 2010. Transport study of nanoparticles for oilfield application. Proceedings of the SPE International Conference on Oilfield Scale, May 26-27, Aberdeen, UK. DOI: 10.2118/131158-MS

Zhang, D.M., S. Fang, A.A. Zakhidov, S.B. Lee and A.E. Aliev et al., 2005. Strong, transparent, multifunctional, carbon nanotube sheets. Science, 309: 1215-1219. DOI: 10.1126/science. 1115311 